\title{
A FACILE SYNTHESIS OF SOME NOVEL 1,3,4-THIADIAZOLES AND PYRIDINES LINKED TO BENZOFURAN AS ANTIMICROBIAL AGENTS.
}

\author{
N. A Mahmoud* \\ Department of Chemistry, Faculty of Science (Girls), Al-Azhar University, Nasr City, Cairo, 11754, Egypt. \\ *Corresponding author. Profnamahmoud@gmail.com
}

\begin{abstract}
In the present study, a new series of benzofuran-integrated 1,3,4-thiadiazoles were prepared starting from pyrazol-4-carboxaldehyde derivatives 3a,b. Thus, reaction of compounds 3a,b with benzyl hydrazine carbodithioate afforded hydrazine carbodithioate derivatives $\mathbf{4 a , b}$. Treatment of compound $\mathbf{4 a}$ with hydrazonoyl halides 5e,f afforded 1,3,4-thiadiazoles $\mathbf{6 a}, \mathbf{b}$, also, the reaction of $\mathbf{4 b}$ with hydrazonoyl halides 5a-d yielded 1,3,4-thiadiazoles 7a-d. on the other hand, new pyridine derivatives can be synthesized via the reaction from the reaction of chalcone $\mathbf{8}$ with different $C$-nucleophiles like ethyl acetoacetate, benzoyl acetonitrile and acetyl acetone afforded pyridines 9-11 derivatives were characterized by complete spectral data and elemental analysis. Some of the newly prepared compounds were investigated against different pathogenic microbes. The results of the antimicrobial activity revealed that all the tested compounds showed no activity except compound $\mathbf{6 a}$ which showed weak activity against all tested microorganisms. Compound $\mathbf{6 b}$ showed weak activity against $B$. subtilis too and compound $7 \mathrm{c}$ revealed intermediate activity against $E$. coli.
\end{abstract}

Key words: 2-Acetyl benzofuran, Thiadiazoles, Pyridines, Hydrazonoyl halides, Antimicrobial activity.

\section{INTRODUCTION}

Benzofuran scaffold is present in many naturally occurring compounds and synthetic materials, recent studies demonstrated that benzofuran derivatives have significant pharmacological properties such as antimicrobial [1-3], antitubercular [4-6], anticonvulsant [7], anti-AChE [8], antiinflammatory [9, 10], antagonistic [11], antioxidant [12, 13], anticancer [14, 15] and anti-TB [16] activities. In addition, they are used as anti-Alzheimer's disease [17-19], antiparasitic [20] and antiviral [21] activities. Also, compounds bearing 1,3,4-thiadiazole moiety possess antimicrobial [22, 23], anticancer [24] and anti-inflammatory [25] activities. Moreover, pyridine is present in many natural products such as vitamins (vitamin $\mathrm{B}_{6}$ ) and alkaloids (trigonelline). and antimicrobial [26] and anticancer [27] activities,

From the above, we try to synthesize some new benzofuran derivatives containing either thiadiazole or pyridine moiety and testing the antimicrobial activity for these derivatives.

\section{RESULTS AND DISCUSSION}

The synthetic pathways for the formation of title compounds were illustrated in schemes 1-3. Thus, condensation of 2-acetyl benzofuran (1) with either phenyl hydrazine or 3-chloro phenyl hydrazine in ethanol catalyzed with acetic acid yielded 1-(1-(benzofuran-2yl)ethylidene)-2-(substituted phenyl hydrazine 2a,b. Vilsmeier- Haack reaction for $\mathbf{2 a , b}$ furnished 3-(benzofuran-2-yl)-1-(substituted phenyl)-1H-pyrazole-4-carbaldehyde 3a,b[28], respectively; (Scheme 1). The IR spectrum of 3b displayed absorption peaks at $1681 \mathrm{~cm}^{-1}$ characteristic to formyl group. Whereas, the ${ }^{1} \mathrm{H}$ NMR spectrum recorded two singlet signals for $\mathrm{CH}$-pyrazole and formyl group at $\delta 8.44$ and $10.24 \mathrm{ppm}$, respectively.

The formyl group in compound $\mathbf{3 a}, \mathbf{b}$ is condensed with benzyl hydrazinecarbodithioate [28] in isopropanol 
under stirring yielded the condensation produce 4a[30], b, respectively (Scheme 1). The IR spectrum of $\mathbf{4 b}$ lakes aldehydic carbonyl group and revealed new peaks assigned to $\mathrm{NH}$ group at $3165 \mathrm{~cm}^{-1}$ and $1319 \mathrm{~cm}^{-1}(\mathrm{C}=\mathrm{S}) .{ }^{1} \mathrm{H}$ NMR spectrum of $\mathbf{4 b}$ assigned four singlet signals at $\delta 4.51,8.73,9.06$ and $13.38 \mathrm{ppm}$ assigned to $\mathrm{SCH}_{2}, \mathrm{CH}=\mathrm{N}, \mathrm{CH}-$ pyrazole and $\mathrm{NH}$ groups, respectively; Scheme 1.

Cyclocondensation of compound $\mathbf{4 a}$ with hydrazonoyl halides 5e,f32-35] in ethanol containing triethylamine as a catalyst furnished 1,3,4-thiadiazoles 6a,b, respectively; (Scheme 2 ). The reaction proceeded via elimination of hydrogen halide and benzyl mercaptant molecule. The structure The structure of 1,3,4thiadiazoles $6 \mathrm{a}, \mathrm{b}$ was assigned through right elemental analysis and spectral data. The infrared spectrum of $\mathbf{6 a , b}$ lakes the $\mathrm{NH}$ function and two showed band for $\mathrm{C}=\mathrm{O}$ at 1734 and $1735 \mathrm{~cm}^{-1}$. Additionally, ${ }^{1} \mathrm{H}$ NMR spectrum of $\mathbf{6 a}$ recorded signals at $\delta 1.50$ and $4.21 \mathrm{ppm}$, corresponds to $\mathrm{CH}_{3}$ (triplet) and $\mathrm{CH}_{2}$<smiles>CC(=O)c1cc2ccccc2o1</smiles>

1
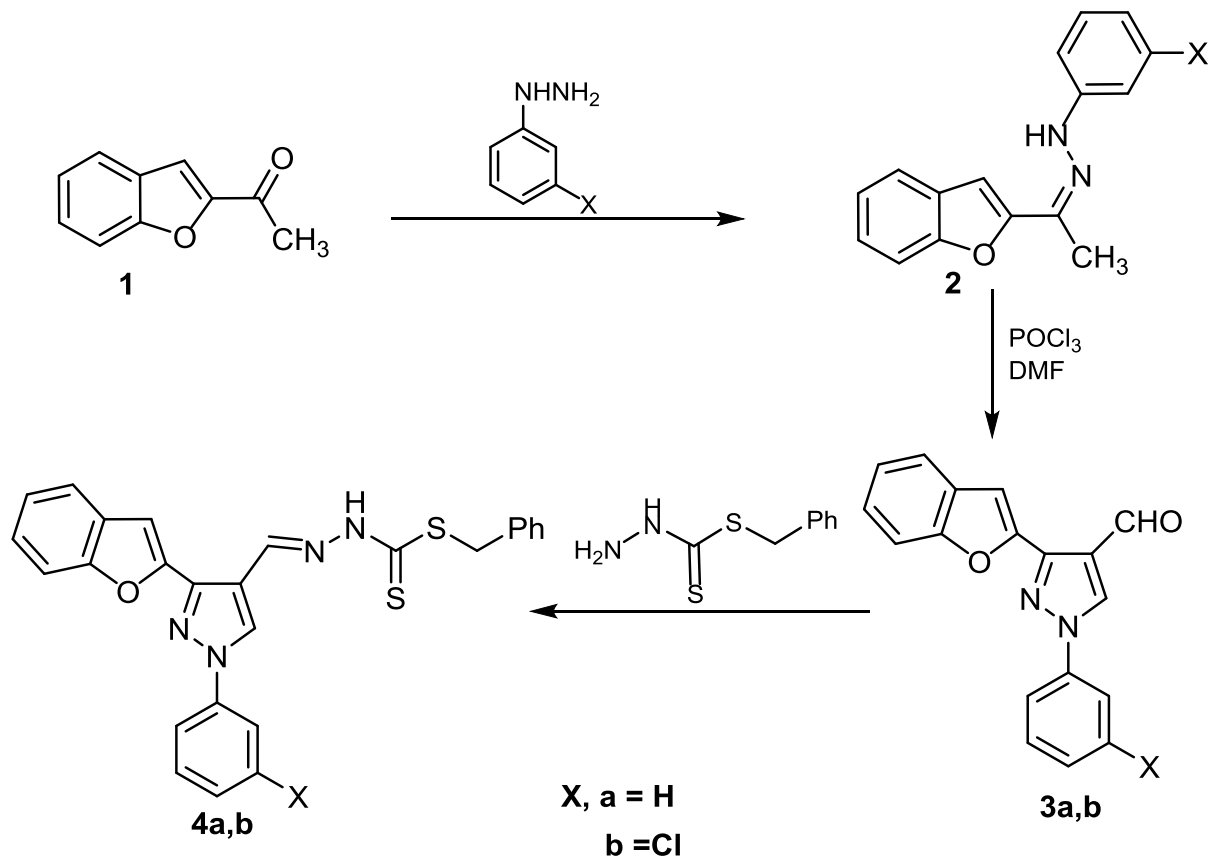<smiles>[X]c1cccc(-n2cc(C)c(-c3cc4ccccc4o3)n2)c1</smiles>

$3 a, b$

Scheme1,Synthesis of some new hydrazones.

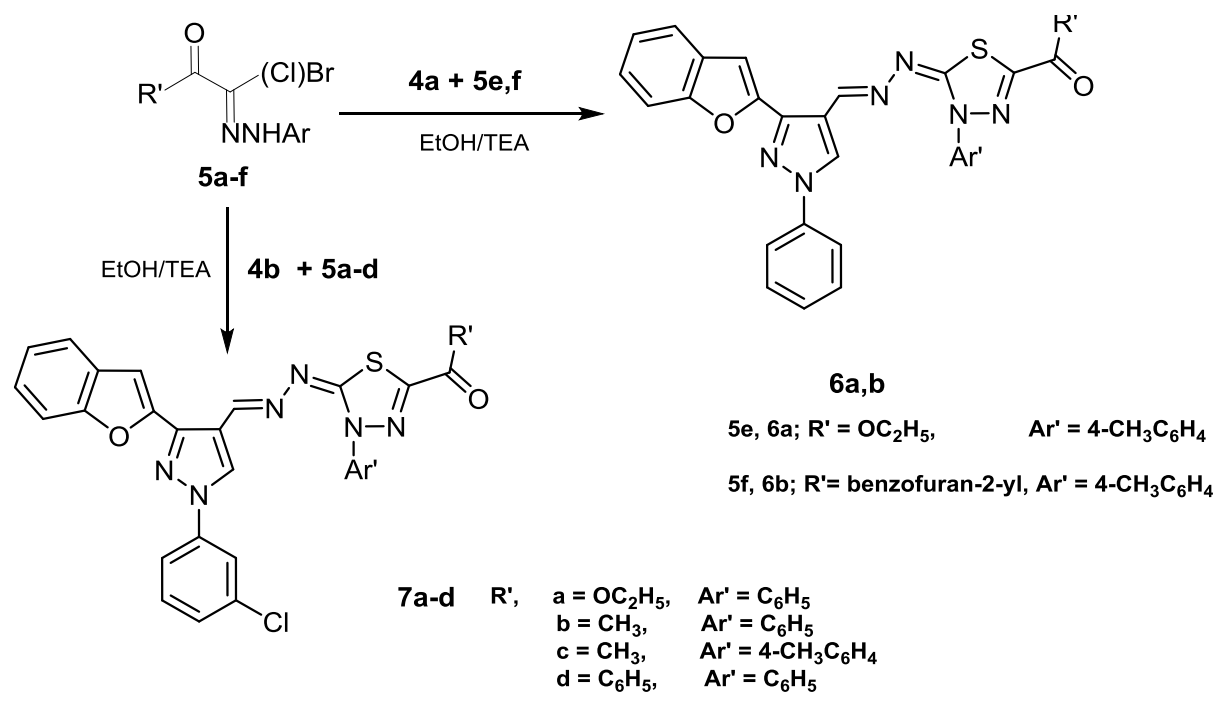

Scheme 2, Synthesis of 1,3,4 thiadiazoles. 
(quartet) of ester group, beside a singlet signal at $\delta 3.27 \mathrm{ppm}$ for methyl protons, two new singlet signals appeared at $\delta \quad \delta 8.62 \& 9.25$ ppm related to $\mathrm{CH}=\mathrm{N}$ and $\mathrm{CH}$-pyrazole, respectively. Similarly, 1,3,4-thiadiazoles 7a-d were accomplished via the reaction of compound 4b with hydrazonoyl halides 5a-d [32-35] under similar conditions. Spectral data and elemental analysis of the prepared compounds approved their structures. For instance, IR spectra of 7a-d revealed absorption peaks for carbonyl group in the range 1732$1651 \mathrm{~cm}^{-1}$. The ${ }^{1} \mathrm{H}$ NMR spectrum of 7a lakes a signal due to $\mathrm{NH}$ group and demonstrated the appearance of triplet and quartet signals for ester group at $\delta 1.31 \& 4.35 \mathrm{ppm}$, besides the signals due to aromatic protons in region $\delta$ $7.30-8.14 \mathrm{ppm}$ and two singlet signals at $\delta 8.67$ and $9.14 \mathrm{ppm}$ due to $\mathrm{CH}=\mathrm{N}$ and $\mathrm{CH}$-pyrazole, respectively.

The ${ }^{1} \mathrm{H}$ NMR spectrum of (7b;DMSO- $\left.d_{6}\right)$ recorded new singlet signal at $\delta 2.77 \mathrm{ppm}$ for acetyl group, protons for aromatic at $\delta$ 7.27$7.82 \mathrm{ppm}$ and at $\delta 8.46 \mathrm{ppm}$ for $\mathrm{CH}=\mathrm{N}$. Whilst, the ${ }^{1} \mathrm{H}$ NMR spectrum of (7c ; DMSO- $\left.d_{6}\right)$ illustrated new singlet signals at 2.40 and 2.66 ppm related to methyl and acetyl proton, consequently, as well as aromatic protons at $\delta$
7.26-8.08 ppm and singlet signals at $\delta 8.45$ ppm related to $\mathrm{CH}=\mathrm{N}$.

Finally, treatment of chalcone $\mathbf{8}$ with different $C$-nucleophile such as ethyl acetoacetate, benzoyl acetonitrile or acetyl acetone in acetic acid in presence of ammonium acetate under reflux afforded pyridines 9-11, respectively (Scheme 3). Spectroscopic data of the synthesized products elucidated their structure. Thus, the IR spectrum of 9 displayed absorption band at $1720 \mathrm{~cm}^{-1}$ corresponds to $\left(\mathrm{C}=\mathrm{O}\right.$ ester). Its ${ }^{1} \mathrm{H}$ NMR spectrum showed triplet signal at $\delta 1.06$ ppm and quartet signal $\delta 4.21 \mathrm{ppm}$ for ester group beside singlet signal at $\delta 2.24 \mathrm{ppm}$ for $\mathrm{CH}_{3}$ group, and at $\delta$ 7.26-7.82 ppm for aromatic protons. Furthermore the mass spectrum of 9 displayed a molecular ion peak at $\mathrm{m} / \mathrm{z} 500\left(\mathrm{M}^{+}\right.$ $+1,1.2)$, 77 (100). Furthermore, in the IR spectrum of $\mathbf{1 0}$ absorption peaks at $2221 \mathrm{~cm}^{-1}$ was observed for cyano group. Its ${ }^{1} \mathrm{H}$ NMR spectrum recorded signals for aromatic protons at $\delta 7.27-8.50 \mathrm{ppm}$. The ${ }^{1} \mathrm{H}$ NMR spectrum 11 revealed singlet signals at $\delta 2.63$ and $2.89 \mathrm{ppm}$ corresponds to $\mathrm{CH}_{3}$ and $\mathrm{COCH}_{3}$ protons, respectively, aromatic protons appeared in region 7.26-8.03 ppm.

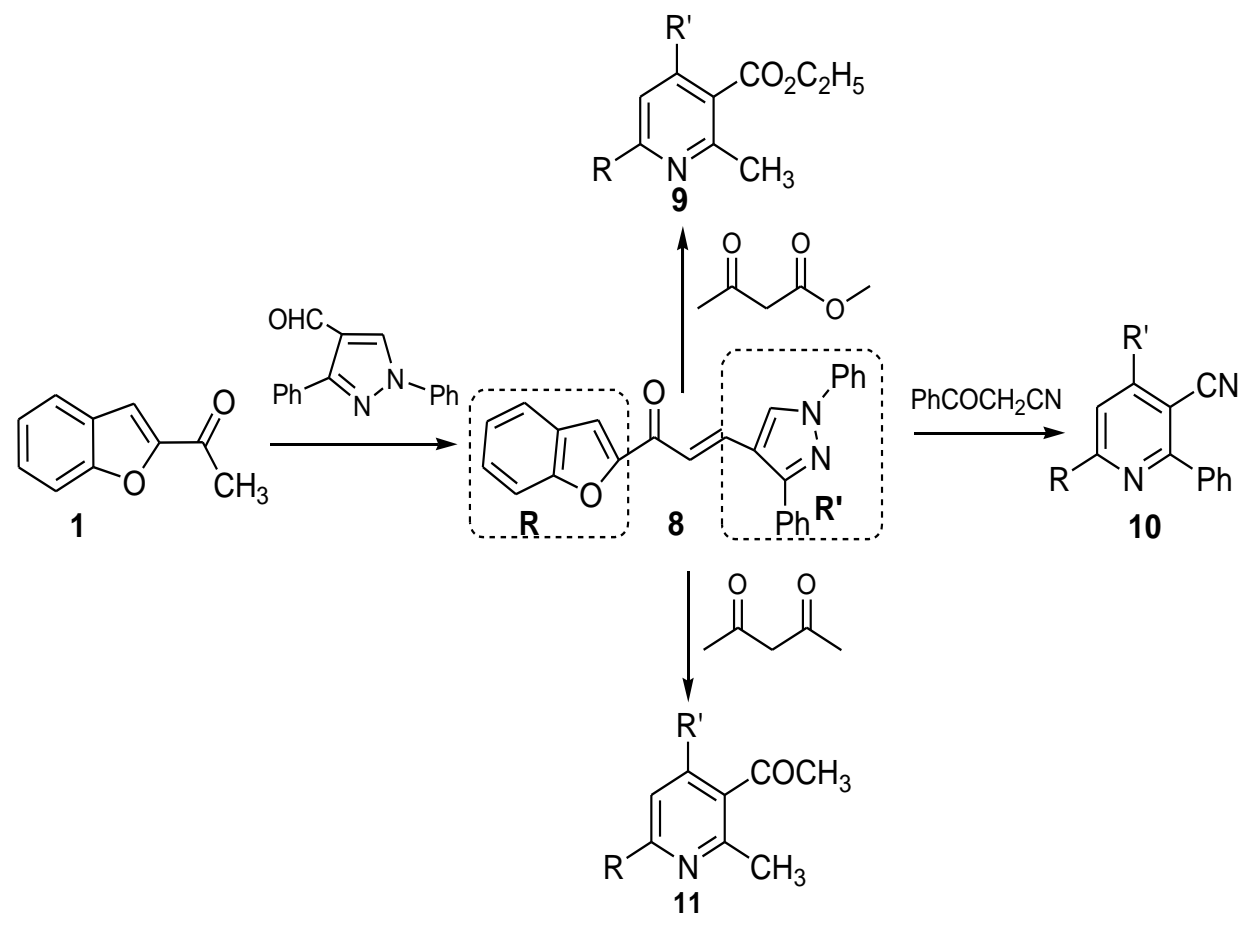




\section{Biological Screening}

Some of newly prepared compounds were investigated against two $(\mathrm{G}+\mathrm{ve})$ bacteria, two (G-ve) bacteria and two fungal species. The screening results revealed that all the tested compounds showed no activity except thiadiazole derivative 6a which showed week activity against all tested microorganisms. Compound 6b was week active against $B$. subtilis and compound $\mathbf{7 c}$ was intermediate active against $E$. coli.

\section{EXPERIMENTAL SECTION}

The melting points of the prepared compounds were measured on an electrothermal apparatus and may be uncorrected. The infrared spectra were determined ( $\mathrm{KBr}$ discs) on a Shimadzu FT-IR 8201 PC spectrophotometer. Mass spectra were determined on Thermo Scientific ISQLT mass spectrometer at the Regional Center for Mycology and Biotechnology, Al-Azhar University. The ${ }^{1} \mathrm{H}$ NMR spectra were performed on a Bruker spectrometer at 400 $\mathrm{MHz}$, TMS was the internal standard.

Compounds 3a [29], 4a [30], 8 [31] and hydrazonoyl halides 5a-f [32-35] were previously prepared.
Synthesis of 3-(benzofuran-2-yl)-1-(3chlorophenyl)-1 $H$-pyrazole-4-carbaldehyde (3b).

To $N, N$-dimethyl formamide $(15 \mathrm{~mL})$, phosphorus oxychloride $(2 \mathrm{~mL})$ was added drop wise at $0-5{ }^{\circ} \mathrm{C}$ with stirring, hydrazone $2 \mathbf{b}$ $(2.50 \mathrm{~g})$ was added very slowly and stirred for $3 \mathrm{~h}$, . The precipitate was filtered, dried and crystallized from DMF yielded compound $\mathbf{3 b}$. White; yield: $91 \%$; mp.: $190-92^{\circ} \mathrm{C}$. FT-IR $\left(\mathrm{KBr}, v \mathrm{~cm}^{-1}\right): 3120,2916,2877(\mathrm{CH}), 1681$ $(\mathrm{C}=\mathrm{O}), 1569(\mathrm{C}=\mathrm{N}) ;{ }^{1} \mathrm{H}$ NMR: $\delta$ 7.35-8.12 (m, 9H, Ar-H), 8.44 (s, 1H, pyrazole ring), 10.24 ppm (s, 1H, CHO). Anal. Calcd for $\mathrm{C}_{18} \mathrm{H}_{11} \mathrm{ClN}_{2} \mathrm{O}_{2}$ (322.75): $\mathrm{C}, 66.99 ; \mathrm{H}, 3.44 ; \mathrm{N}$, 8.68. Found: C, 66.91; H, 3.54; N, 8.60.

Synthesis of benzyl-2-(3-(benzofuran-2-yl)-1(3-chlorophenyl)-1H-pyrazol-4-ylmethylene)-hydrazine carbodithioate (4b)

Equimolar amounts of $\mathbf{3 b}$ and benzyl hydrazine carbodithioate in isopropanol were stirred for $30 \mathrm{~min}$. The solid formed was collected and crystallized from DMF to give 4b. Paige; yield: $82 \%$; mp.: 230-32 ${ }^{\circ} \mathrm{C}$; FT-IR $\left(\mathrm{KBr}, v \mathrm{~cm}^{-1}\right)$ : $3165(\mathrm{NH}), 3062,2916(\mathrm{CH})$, $1589(\mathrm{C}=\mathrm{N}), 1319(\mathrm{C}=\mathrm{S}) ;{ }^{1} \mathrm{H}$ NMR: $\delta 4.51(\mathrm{~s}$, $\left.2 \mathrm{H}, \mathrm{SCH}_{2}\right), 7.25-7.47(\mathrm{~m}, 10 \mathrm{H}), 7.53(\mathrm{t}, 1 \mathrm{H})$,

Table1: Response of various microorganisms to some of synthesized compounds.

\begin{tabular}{|c|c|c|c|c|c|c|}
\hline \multirow{3}{*}{$\begin{array}{l}\text { Compd. } \\
\text { NO. }\end{array}$} & \multicolumn{6}{|c|}{ Mean* of zone diameter nearest whole mm. } \\
\hline & \multicolumn{2}{|c|}{$\begin{array}{c}\text { Gram - positive } \\
\text { bacteria }\end{array}$} & \multicolumn{2}{|c|}{ Gram - negative bacteria } & \multicolumn{2}{|c|}{ Yeasts and Fungi** } \\
\hline & S.aureus & B. subtilis & S. typhimurium & E. coli & C. albicans & A. fumigatus \\
\hline $\mathbf{3 b}$ & - & - & - & - & - & - \\
\hline $4 b$ & - & - & - & - & - & - \\
\hline $\mathbf{6 a}$ & 10 & 10 & 11 & 13 & - & 11 \\
\hline $6 \mathbf{b}$ & - & 11 & - & - & - & - \\
\hline $7 \mathbf{a}$ & - & - & - & - & - & - \\
\hline 7c & - & - & - & 15 & - & - \\
\hline $7 d$ & - & - & - & - & - & - \\
\hline 11 & - & - & - & - & - & - \\
\hline Control \# & 35 & 35 & 36 & 38 & 35 & 37 \\
\hline
\end{tabular}

* = Calculate from 3 values; $* *=$ identified on the basis of routine cultural, morphological and microscopical characteristics, - = No effect, \#: Chloroamphencol(Gram+ve),Cephalothin(Gramve),Cyclohexamide in case of fungi. 
$7.64(\mathrm{~d}, 1 \mathrm{H}, J=8 \mathrm{~Hz}), 7.79(\mathrm{~d}, 1 \mathrm{H}, J=8 \mathrm{~Hz})$, $7.99(\mathrm{~d}, 1 \mathrm{H}, J=8 \mathrm{~Hz}), \quad 8.73(\mathrm{~s}, 1 \mathrm{H}, \mathrm{CH}=\mathrm{N})$, 9.06 (s, 1H, CH-pyrazole), $13.38 \mathrm{ppm}(\mathrm{s}, 1 \mathrm{H}$, $\mathrm{NH})$. Anal. Calcd. For $\mathrm{C}_{26} \mathrm{H}_{19} \mathrm{ClN}_{4} \mathrm{OS}_{2}$ (503.04): C, 62.08; H, 3.81; N, 11.14. Found: C, 62.15; $\mathrm{H}, 3.89 ; \mathrm{N}, 11.20$.

Synthesis of 1,3,4-thiadiazoles 6a,b and 7a-d.

To stirred solution of $\mathbf{4 a}$ or $\mathbf{4 b}(5 \mathrm{mmol})$ in $20 \mathrm{~mL}$ ethanol having 0.01 mole trimethylamine as catalyst, hydrazonoyl halides 5a-f $(5 \mathrm{mmol})$ was added separately. The precipitate was filtered and crystallized from a proper solvent and gave thiadiazoles $\mathbf{6 a}, \mathbf{b}$ and 7a-d, respectively.

2-(Ethoxycarbonyl)-5-(2-((3-(benzofuran-2yl)-1-phenyl-1 $H$-pyrazol-4yl)methylene)hydrazono)-4,5-dihydro-4-(4methylphenyl)-1,3,4-thiadiazole (6a).

Yellow (DMF), yield: 80\%; mp.: 210$11^{\circ} \mathrm{C}$; FT-IR $\left(\mathrm{KBr}, v \mathrm{~cm}^{-1}\right)$ : 3039, $2978(\mathrm{CH})$, $1735(\mathrm{C}=\mathrm{O}), 1597(\mathrm{C}=\mathrm{N}) ;{ }^{1} \mathrm{H}$ NMR: $\delta 1.50(\mathrm{t}$, $\left.3 \mathrm{H}, J=7 \mathrm{~Hz}, \mathrm{CH}_{3}\right), 3.27$ (s, 3H, $\left.\mathrm{CH}_{3}\right), 4.21$ (q, $\left.2 \mathrm{H}, J=7 \mathrm{~Hz}, \mathrm{CH}_{2}\right), 7.33-7.71(\mathrm{~m}, 10 \mathrm{H}, \mathrm{Ar}-\mathrm{H})$, $7.75(\mathrm{~d}, 2 \mathrm{H}, \mathrm{J}=8 \mathrm{~Hz}), 7.79(\mathrm{~d}, 2 \mathrm{H}, \mathrm{J}=8 \mathrm{~Hz})$, $8.62(\mathrm{~s}, 1 \mathrm{H}, \mathrm{CH}=\mathrm{N}), 9.25 \mathrm{ppm}(\mathrm{s}, 1 \mathrm{H}, \mathrm{CH}-$ pyrazole). Anal Calcd. for $\mathrm{C}_{30} \mathrm{H}_{24} \mathrm{~N}_{6} \mathrm{O}_{3} \mathrm{~S}$ (548.16): C, 65.68; H, 4.41; N, 15.32; . Found: C, 65.75; H, 4.50; N, 15.40.

2-(Benzofuran-2-yl-carbonyl)-5-(2-((3(benzofuran-2-yl)-1-phenyl-1H-pyrazol-4yl)methylene)hydrazono)-4,5-dihydro-4-(4methyl phenyl)-1,3,4-thiadiazole (6b)

Red (DMF), yield: $82 \%$; mp.: $230-31^{\circ} \mathrm{C}$; FT-IR $\left(\mathrm{KBr}, v \mathrm{~cm}^{-1}\right): 3101,2978(\mathrm{CH}), 1734$ $(\mathrm{C}=\mathrm{O}), 1597 \quad(\mathrm{C}=\mathrm{N}) ;{ }^{1} \mathrm{H}$ NMR: $\delta 4.51(\mathrm{~s}, 3 \mathrm{H}$, $\left.\mathrm{CH}_{3}\right), 7.23-8.02(\mathrm{~m}, 19 \mathrm{H}, \mathrm{Ar}-\mathrm{H}), 8.73(\mathrm{~s}, 1 \mathrm{H}$, $\mathrm{CH}=\mathrm{N}), 9.04$ (s, 1H, CH-pyrazole). Anal Calcd. for $\mathrm{C}_{36} \mathrm{H}_{24} \mathrm{~N}_{6} \mathrm{O}_{3} \mathrm{~S}$ (620.16): C, 69.66; H, 3.90; N, 13.54. Found: C, 69.74; H, 3.99; N, 13.45.

2-(Ethoxycarbonyl)-5-(2-((3-(benzofuran-2yl)-1-(3-chlorophenyl)-1H-pyrazol-4yl)methylene)hydrazono)-4,5-dihydro-4phenyl-1,3,4-thiadiazole (7a).

Yellow (DMF), yield: $85 \%$; mp $207-8^{\circ} \mathrm{C}$; FT-IR $\left(\mathrm{KBr}, v \mathrm{~cm}^{-1}\right)$ : 3059, $2932(\mathrm{CH}), 1732$ $(\mathrm{C}=\mathrm{O}), 1597(\mathrm{C}=\mathrm{N}) ;{ }^{1} \mathrm{H}$ NMR: $\delta 1.31(\mathrm{t}, 3 \mathrm{H}, J$ $\left.=7 \mathrm{~Hz}, \mathrm{CH}_{3}\right), 4.35\left(\mathrm{q}, 2 \mathrm{H}, J=7 \mathrm{~Hz}, \mathrm{CH}_{2}\right), 7.30-$ $8.14(\mathrm{~m}, 13 \mathrm{H}, \mathrm{ArH}), 8.67(\mathrm{~s}, 1 \mathrm{H}, \mathrm{CH}=\mathrm{N}), 9.14$ ppm (s, 1H, CH-pyrazole). Anal Calcd. for $\mathrm{C}_{29} \mathrm{H}_{21} \mathrm{ClN}_{6} \mathrm{O}_{3} \mathrm{~S}(569.03): \mathrm{C}, 61.21 ; \mathrm{H}, 3.72 ; \mathrm{N}$, 14.77. Found: C, 61.11; H, 3.63; N, 14.70.

2-Acetyl-5-(2-((3-(benzofuran-2-yl)-1-(3chlorophenyl)-1 $H$-pyrazol-4yl)methylene)hydrazono)-4,5-dihydro-4phenyl-1,3,4-thiadiazole (7b)

Yellow (EtOH); yield: 83\%; mp.: 110$12^{\circ} \mathrm{C}$; FT-IR $\left(\mathrm{KBr}, v \mathrm{~cm}^{-1}\right): 3062,2970(\mathrm{CH})$, $1681(\mathrm{C}=\mathrm{O}), 1597(\mathrm{C}=\mathrm{N}) ;{ }^{1} \mathrm{H}$ NMR: $\delta 2.77(\mathrm{~s}$ $\left.3 \mathrm{H}, \mathrm{COCH}_{3}\right), 7.27-7.59(\mathrm{~m}, 14 \mathrm{H}, \mathrm{ArH}), 7.82$ $(\mathrm{d}, 1 \mathrm{H}, J=8 \mathrm{~Hz}), 8.46 \mathrm{ppm}(\mathrm{s}, 1 \mathrm{H}, \mathrm{CH}=\mathrm{N})$. Anal Calcd. for $\mathrm{C}_{28} \mathrm{H}_{19} \mathrm{ClN}_{6} \mathrm{O}_{2} \mathrm{~S}(539.01)$ : $\mathrm{C}$, 62.39; H, 3.55; N, 15.59. Found: C, 62.29; H, $3.65 ; \mathrm{N}, 15.50$.

2-Acetyl-5-(2-((3-(benzofuran-2-yl)-1-(3chlorophenyl)-1 $H$-pyrazol-4yl)methylene)hydrazono)-4,5-dihydro-4-(4methylphenyl)-1,3,4-thiadiazole (7c)

Red (DMF), yield: 83\%; mp.: 265-67 ${ }^{\circ}$; FT-IR $\left(\mathrm{KBr}, v \mathrm{~cm}^{-1}\right)$ : 3068, $2927(\mathrm{CH}), 1666$ $(\mathrm{C}=\mathrm{O}), 1593(\mathrm{C}=\mathrm{N}) ;{ }^{1} \mathrm{H}$ NMR: $\delta 2.40(\mathrm{~s}, 3 \mathrm{H}$, $\left.\mathrm{CH}_{3}\right), 2.66$ (s $\left.3 \mathrm{H}, \mathrm{COCH}_{3}\right), 7.26-7.34(\mathrm{~m}, 3 \mathrm{H}$, $\mathrm{ArH})$, 7.61-7.78 (m, 7H, ArH), $7.92(\mathrm{~d}, 2 \mathrm{H}, J=$ $8 \mathrm{~Hz}), 8.08(\mathrm{~d}, 2 \mathrm{H}, J=8 \mathrm{~Hz}), 8.45 \mathrm{ppm}(\mathrm{s}, 1 \mathrm{H}$, $\mathrm{CH}=\mathrm{N})$. Anal Calcd. for $\mathrm{C}_{29} \mathrm{H}_{21} \mathrm{ClN}_{6} \mathrm{O}_{2} \mathrm{~S}$ (553.03): C, 62.98; H, 3.83; N, 15.20. Found: C, 62.88; H, 3.90; N, 15.27.

2-Benzoyl-5-(2-((3-(benzofuran-2-yl)-1-(3chlorophenyl)-1 $H$-pyrazol-4yl)methylene)hydrazono)-4,5-dihydro-4phenyl-1,3,4-thiadiazole (7d)

Red (DMF), yield: $84 \%$; mp.:180-81 ${ }^{\circ} \mathrm{C}$; FT-IR $\left(\mathrm{KBr}, v \mathrm{~cm}^{-1}\right)$ : 3050, $2935(\mathrm{CH}), 1651$ $(\mathrm{C}=\mathrm{O}), 1620(\mathrm{C}=\mathrm{N}) ;{ }^{1} \mathrm{H}$ NMR: $\delta$ 7.31-8.24 (m, 19H, ArH), 8.45 (s, 1H, CH=N), 9.16 ppm (s, 1H, CH-pyrazole). Anal Calcd. for $\mathrm{C}_{33} \mathrm{H}_{21} \mathrm{ClN}_{6} \mathrm{O}_{2} \mathrm{~S}$ (601.08): C, 65.94; H, 3.52; N, 13.98. Found: C, 65.86; H, 3.60; N, 13.90 .

\section{Synthesis of pyridine derivatives 9-11.}

To a solution of $\mathbf{8}(5 \mathrm{mmol})$ in acetic acid (20 $\mathrm{ml})$ having ammonium acetate $(10 \mathrm{mmol})$, ethyl acetoacetate, benzoyl acetonitrile and acetyl acetone $(5 \mathrm{mmol})$ was added separately. 
The mixture were boiled for $3 \mathrm{~h}$. then allowed to cool. The precipitate was collected and crystallized from a proper solvent to give 9-11, respectively.

\section{Ethyl 4-(1,3-diphenyl-1H-pyrazol-4-yl)-2- methyl-6-(benzofuran-2-yl) pyrid- ine-3- carboxylate (9).}

White (EtOH); Yield: 85\%; mp.: 159-60 ${ }^{\circ} \mathrm{C}$. FT-IR (KBr, $\left.v, \mathrm{~cm}^{-1}\right): 3058,2962,2873$ $(\mathrm{CH}), 1720(\mathrm{C}=\mathrm{O}), 1639(\mathrm{C}=\mathrm{N}) ;{ }^{1} \mathrm{H}$ NMR: $\delta$ $1.06\left(\mathrm{t}, 3 \mathrm{H}, J=7.5 \mathrm{~Hz}, \mathrm{CH}_{3}\right), 2.24(\mathrm{~s}, 3 \mathrm{H}$, $\mathrm{CH}_{3}$ ), 4.21 (q, $2 \mathrm{H}, J=7.5 \mathrm{~Hz}, \mathrm{CH}_{2}$ ), 7.26-7.44 (m, 12H, ArH's), 7.67 (d, 2H, $J=8 \mathrm{~Hz}), 7.75$ (s, 1H), $7.82 \mathrm{ppm}(\mathrm{d}, 2 \mathrm{H}, J=8 \mathrm{~Hz}) ; \mathrm{MS} \mathrm{m} / \mathrm{z}$ (\%): $500\left(\mathrm{M}^{+}+1,1.2\right), 77$ (100). Anal. Calcd for $\mathrm{C}_{32} \mathrm{H}_{25} \mathrm{~N}_{3} \mathrm{O}_{3}$ (499.56): C, 76.94; $\mathrm{H}, 5.04 ; \mathrm{N}$, 8.41. Found: C, 76.84; H, 5.14; N, 8.31.

\section{6-(Benzofuran-2-yl)-4-(1,3-diphenyl-1H- pyrazol-4-yl)-2-phenyl pyridine-3- carbonitrile (10).}

Yellow $(\mathrm{AcOH})$; yield: 80\%; mp.: 210$11^{\circ} \mathrm{C}$; FTIR (KBr, $\left.v, \mathrm{~cm}^{-1}\right): 3055(\mathrm{CH}), 2221$ $(\mathrm{CN}), 1596(\mathrm{C}=\mathrm{N}) ;{ }^{1} \mathrm{H}$ NMR: $\delta$ 7.27-7.67 $(\mathrm{m}$, $16 \mathrm{H}), 7.81(\mathrm{~s}, 1 \mathrm{H}), 7.85(\mathrm{~d}, 2 \mathrm{H}, J=8 \mathrm{~Hz}), 7.97$ $(\mathrm{t}, 2 \mathrm{H}, J=7.6 \mathrm{~Hz}), 8.50 \mathrm{ppm}(\mathrm{s}, 1 \mathrm{H})$. Anal. Calcd for $\mathrm{C}_{35} \mathrm{H}_{22} \mathrm{~N}_{4} \mathrm{O}$ (514.58): C, 81.69; H, 4.31 ; N, 10.89. Found: C, 81.79; H, 4.41; N, 10.80 .

\section{1-(6-(Benzofuran-2-yl)-2-methyl-4-(1,3- diphenyl-1H-pyrazol-4-yl)pyridin-3- yl)ethanone (11).}

Brown (AcOH); yield: 80\%; mp: 220$21^{\circ} \mathrm{C}$; FTIR (KBr, v, $\left.\mathrm{cm}^{-1}\right): 3055(\mathrm{CH}), 1680$ $(\mathrm{C}=\mathrm{O}), 1596(\mathrm{C}=\mathrm{N})$; ${ }^{1} \mathrm{H}$ NMR: $\delta 2.63(\mathrm{~s}, 3 \mathrm{H}$, $\left.\mathrm{CH}_{3}\right), 2.89\left(\mathrm{~s}, 3 \mathrm{H}, \mathrm{COCH}_{3}\right), 7.26-7.42(\mathrm{~m}$, $13 \mathrm{H}), 7.46(\mathrm{t}, 1 \mathrm{H}, J=7.6 \mathrm{~Hz}), 7.58(\mathrm{~d}, 1 \mathrm{H}, J=$ $8 \mathrm{~Hz}), 7.68(\mathrm{~d}, 1 \mathrm{H}, J=8 \mathrm{~Hz}), 8.03 \mathrm{ppm}(\mathrm{s}, 1 \mathrm{H})$. Anal. Calcd for $\mathrm{C}_{31} \mathrm{H}_{23} \mathrm{~N}_{3} \mathrm{O}_{2}$ (469.53): C, 79.30; H, 4.94; N, 8.95. Found: , 79.20; H, 4.87; N, 8.86 .

\section{Biological Screening}

Investigation of the antimicrobial activity of some prepared compounds were carried out using standardized disc - agar diffusion method [36].

\section{Conflict of interest}

There are no conflicts to declare

\section{CONCLUSIONS}

In this research, new series of thiadiazoles and pyridines (6a,b), (7a-e) and (9-11) were investigated for their antimicrobial activity against different pathogenic microorganisms. The results revealed that all the tested compounds showed no activity except compound 6a which showed week activity against all tested microorganisms. Compound 6b showed week active against $B$. subtilis and compound 7c showed intermediate active against $E$. coli.

\section{REFERENCES}

[1] Abdel-Aziem, A., Baaiu, B. S.; Abdelhamid, A. O. (2017): J. Heterocyclic Chem. 54, 3471.

[2] Abdel-Aziem, A. (2015): Int J Pharm Pharm Sci., 7, 61.

[3] Abdel-Aziem, A., Abdelhamid, A. O. (2013): Int J Adv Res., 1, 717.

[4] Manna, K., Agrawal, Y. K. (2010): Eur J Med Chem., 45, 3831.

[5] Trapero, A., Pacitto, A., Singh, V., Sabbah, M., Coyne, A. G., Mizrahi, V., Blundell, T. L., Ascher, D. B., Abell, C. (2018): J. Med. Chem., $61,2806$.

[6] Zhang, W., Lun, S., Wang, S. H., Jiang, X. W., Yang, F., Tang, J., Manson, A. L., Earl, A. M., Gunosewoyo, H., Bishai, W.R., Yu, L.F.(2018): J. Med. Chem., 61, 791.

[7] Kamal, M., Shakya, A. K.(2012): Int J Biomed \& Adv Res., 3, 233.

[8] Xiang, Z., Miao, L., Wang, X.-B., Wang, T., Kong, L.-Y.(2010): Molecules., 15, 8593.

[9] Lakshminarayana, K., Wari, U. G., Kendri, S. S. (2016): Int J Bas Clin Pharmacol., 5, 239.

[10] Timonen, J., Vuolteenaho, K., Leppänen, T., Nieminen, R., Moilanen, E., Aulaskari, P., Jänis, J. (2015): J Heterocyclic Chem., 52, 1286.

[11] Jain, S. K., Sinha, P. (2011): Der Pharma Chemica, 3, 572.

[12] Sajjadi-Ghotbabadi, H., Javanshir, S., RostamiCharati, F.(2017): J Heterocyclic Chem., 54, 979.

[13] Rindhe, S. S., Rode, M. A., Karale, B.K. (2010): Ind. J. Pharma.1 Sci.,72(2):231.

[14] Abdel-Aziem, A. J. (2017): Heterocyclic Chem., 54, 2985.

[15] Napiórkowska, M., Cieślak, M., KaźmierczakBarańska, J., Królewska-Golińska, K., Nawrot, B.(2019): Molecules, 24,1529. 
[16] Thorata, B. R., Nazirkara, B., Thorat, V. B., Mandewalea, M., Nagarsekara, A., Yamgarc, R. S. (2016): J Chem Sci Photon., 110, 279.

[17] Cabrera-PardoJorge, J. R., Fuentealba, J., Gavilán, J., Cajas, D., Becerra, J., Napiórkowska, M. (2020). Front Pharmacol.,11,709.

[18] Hiremathad, A., Chand, K.,Tolayan, L., Rajeshwari, Keri, R. S., Esteves, A. R., Cardoso, S. M., Chaves, S., Santos, M. A. (2018): J. Inorg. Biochem., 179, 82.

[19] Deepti, G., Amandeep, K., Bhupesh, G. (2018): ChemMedChem., 13, 1275.

[20] Khanam, H., Uzzaman, S.(2015): Eur. J. Med. Chem., 97, 483.

[21] Zhong, M., Peng, E., Huang, N., Huang, Q., Quq, A., Lau, M., Colonno, R., Li, L. (2018): Bioorg Med Chem Lett., 28, 963.

[22] Serban, G., Stanasel, O.,Serban, E., Bota, S. (2018): Drug Des. Devel. Ther., 12, 1545.

[23] Abo-Bakr, A. M., Hashem, H.E. (2019): J. Heterocycl. Chem., 56, 1038.

[24] Altintop, M. D., Sever, B., Ozdemir, A., Llgin, S., Atl,i O., Turan-Zitouni, G., Kaplancikli, Z.A. (2018): Anticancer Agents Med Chem., $18(11), 1606$.

[25] Popov, N. S., Demidova, M. A., Malygin, A. S. (2018): Res. Results Pharmacolo., 4(2), 27.

[26] Radwan, M. A. A., Alshubramy, M. A., AbdelMotaal, M., Hemdan, B. A., El-Kady, D. S. (2020): Bioorg. Chem.,14(1) 40.

[27] Androutsopoulos, V. P., Spandidos, D. A. (2017): Onclogy Reports., 38, 519.

[28] Klayman, D.L., Bartosevich, J. F., Griffin, T. S., Mason, C. J., Scovill, J. P. (1979): J Med Chem., 22(7), 855.

[29] Kumar, D. B., Drakash, G. K., Kumaraswamy, M. N., Nandeshwarappa, B. P., Sherigara, B. S., Mahadevan, K. M. (2007): Ind J Chem., $46 \mathrm{~B}(2), 336$.

[30] Abdel-Aziem, A. (2015): Int J Pharm Pharm Sci., 7(1), 61.

[31] Abdel-Aziem, A., Abdelhamid, A. O. (2013): Int J Adv Res., 1(9), 717.

[32] Asiri, A. M., Al-youbi, A. O., Zayed, M. E. M., Ng, S.W. (2011): Acta. Cryst Sec E Struct. Rep. 67(Pt 8):01962.

[33] Eweiss, N. F., Osman, A. (1980): J Heterocycl Chem. 17, 1713.

[34] Abdelamid, A. O., El-Shiatey, F. H. H. (1988): Phosphorus Sulfur Silicon Relat. Elem., 39, 45.

[35] Hassaneen, H. M., Shawali, A. S., Elwan, N. M., Abounada, N. M. (1992): Sulfur Lett., 13, 273.

[36] Bauer, A. W., Kirby, W. W. M.;,Sherris, J. C. (1966): Turck M. Am J Clin Pathol., 45, 493.

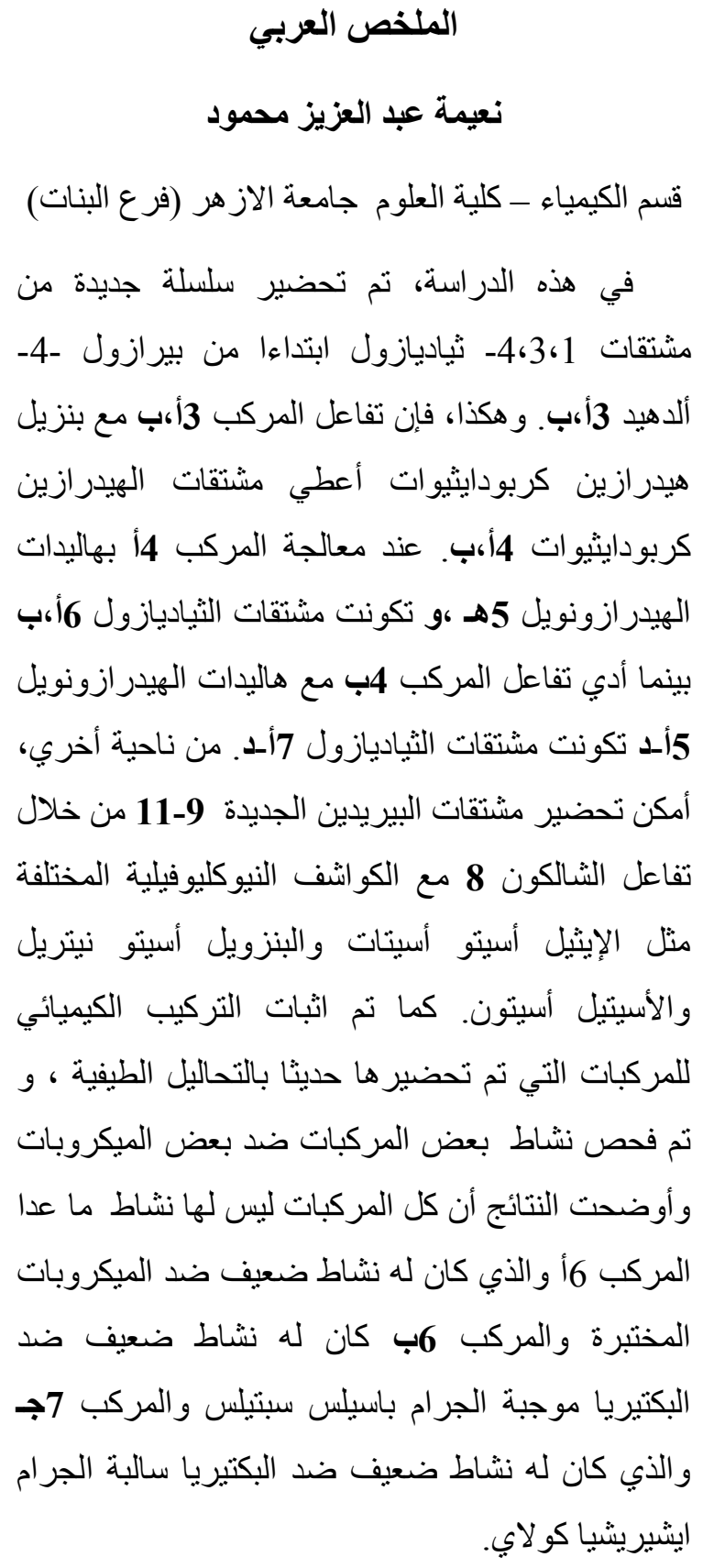

